\title{
Comparative efficacy and acceptability of seven augmentation agents for treatment-resistant depression: A multiple-treatments meta-analysis
}

\author{
X Wang, MMed; S Huang, MMed; H B Qi, PhD \\ Department of Obstetrics and Gynecology, The First Affiliated Hospital of Chongqing Medical University, Chongqing, China \\ Corresponding author: H B Qi (keke_Ou@163.com)
}

Background. Treatment-resistant depression (TRD) is a therapeutic challenge for clinicians. Augmentation pharmacotherapy is effective for TRD, but it is still unclear which augmentation agent is most efficacious.

Objective. To assess the effects of seven augmentation agents on TRD.

Methods. We did a multiple-treatments meta-analysis, accounting for both direct and indirect comparisons. PubMed, the Center for Clinical and Translational Research, Web of Science, Embase, CBM-disc, the Chinese National Knowledge Infrastructure and relevant websites (up to August 2013) were searched for randomised controlled trials (RCTs) about augmentation agents. The following terms were used: 'potentiation', 'augmentation', and 'adjunct' paired with 'depression' and 'resistant depression'. No language limitation was imposed.

Results. We systematically reviewed 12 RCTs (1 936 participants), which included seven augmentation agents: lithium, tricyclic antidepressants (TCAs), atypical antipsychotics (AAPs), antiepileptic drugs (AEDs), buspirone, cognitive behaviour therapy (CBT) and tri-iodothyronine (T3). The results revealed that T3 was more efficacious than lithium, TCAs, AAPs, AEDs, buspirone and CBT with odds ratios (ORs) of 1.58, 1.56, 1.51, 1.47, 1.77 and 1.25, respectively. ORs favoured CBT compared with lithium, TCAs, AAPs, AEDs and buspirone. Buspirone was the least efficacious of all the other augmentation agents tested. AAPs were significantly more acceptable than lithium, and CBT more than buspirone. T3 was slightly more acceptable than lithium, and CBT more than AAPs.

Conclusion. T3 as an augmentation agent should be a clinician's first consideration instead of lithium in acute treatment for TRD. CBT might be a good augmentation agent in some communities. Buspirone should be a final option as an augmentation agent. Further research is needed, such as a well-designed, large-scale controlled trial, to support and draw definite conclusions.

S Afr J Psych 2014;20(3):71-76. DOI:10.7196/SAJP.530 


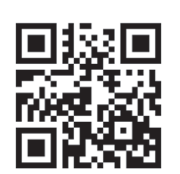

It is forecast that depression will be second only to heart disease as a cause of global disability by $2020 .{ }^{[1]}$ In the past 20 years, available drugs for the treatment of depression have proliferated, many of which are structurally related and share similar putative mechanisms of action. ${ }^{[2]}$ New-generation antidepressant drugs, including serotonin selective reuptake inhibitors and serotonin norepinephrine reuptake inhibitors, have gained wide acceptance, primarily because of their relative safety. ${ }^{[3]}$ However, even with effective pharmacotherapeutic strategies, $\sim 40 \%$ of depressed patients show only partial or non-response to the usual recommended dose of antidepressants, and may be described as treatment-resistant depression (TRD) patients. ${ }^{[4]}$

Sustained remission is the ultimate goal of any antidepressant treatment. For those patients who do not reach remission after the first adequate antidepressant trial, several so-called second-step strategies have been proposed, such as: ( $i$ ) augmentation therapy, ( $i$ ) increasing antidepressant dose, (iii) switching to a different class of antidepressant, (iv) combining two antidepressants. ${ }^{[5]}$ Augmentation pharmacotherapy refers to the addition of drugs that are not standard antidepressants in order to enhance the antidepressive effect of a classic antidepressant drug. ${ }^{[6]}$ These strategies are commonly used by clinicians caring for depressed patients. ${ }^{[7]}$ Two augmentation methods have been studied, namely augmentation treatment at the onset of treatment, or following the failed use of antidepressant monotherapy. The latter approach is used by most of the augmentation studies. ${ }^{[8]}$

Hitherto, many potential agents have been tested for their augmentation effects in clinical trials on TRD. ${ }^{[9]}$ The objective of this review is to integrate the efficacy data of seven kinds of augmentation therapies by multiple-treatments meta-analysis, and provide a clinically useful summary that can be used to guide treatment decisions.

\section{Method}

\section{Study selection}

A comprehensive literature search on randomised controlled trials (RCTs) involving antidepressant augmentation for TRD was first conducted through the major scientific and medical databases, including international databases (PubMed, the Center for Clinical and Translational Research, Web of Science, Embase, EAGLE, National Technical Information Service), two Chinese databases (CBM-disc, Chinese National Knowledge Infrastructure), and relevant websites (Current Controlled Trials, Clinical Trials, International Clinical Trials Registry) up until August 2013. The following terms were used: 'potentiation', 'augmentation', and 'adjunct' paired with 'depression' and 'resistant depression'. There was no language and year of publication limitation. To avoid omitting relevant trials, conference summaries and articles identified from reference lists of previous reviews were checked.

We included RCTs involving antidepressant augmentation for TRD in acute-phase treatment. TRD was defined as the failure of at least one adequate trial of one major class of antidepressant. ${ }^{[10]} \mathrm{We}$ excluded the following studies: those that included patients who had not previously completed an adequate trial of an antidepressant (e.g. because of side-effects); those that compared an antidepressant with placebo; those that included women with post-partum depression; ${ }^{[11]}$ and single case reports, dissertations and meeting abstracts. Bipolar depression was not focused on in this review.

\section{Data extraction}

Two reviewers within the reviewing team independently screened all candidate studies and extracted data. Any disagreements were resolved by discussion within the research team. For data that could not be directly abstracted, the corresponding author was e-mailed or other studies citing the candidate study were obtained. Data retrieved from the candidate studies included the first author, publication year, country, study design, participant characteristics and outcomes (odds of response and drop-out). Response was defined as an absolute Hamilton Depression Rating Scale (HDRS) score or MontgomeryAsberg Depression Scale score reduced by at least 50\% from baseline score, or improvement on the Clinical Global Impression scale by the end of treatment. If all three rating scales were used to evaluate the outcome, we selected the HDRS results. At the end of augmentation treatment, odds of response was the primary outcome, and drop-out was a secondary outcome.

\section{Statistical analysis}

The dichotomous primary outcome was chosen mainly for clinical reasons. In order to make the interpretation of results easier for clinicians, ${ }^{[12]}$ response rates instead of a continuous symptom score were used for efficacy analysis. If baseline scores, standard deviations (SDs) and endpoint means were provided instead of dichotomous efficacy outcomes, we estimated the number of patients responding with a validated imputation method. ${ }^{[13]}$ To carry out a clinically sound analysis, we used a worst-case scenario analysis of drop-out patients, assuming that all those patients did not respond to treatment. ${ }^{[2]}$

First, we did a meta-analysis of augmentation agents that had direct comparison with a random-effect model, using RevMan 5.0 (The Nordic Cochrane Centre, The Cochrane Collaboration, Copenhagen) software. For each analysis, we assessed heterogeneity using the $\chi^{2}$-based $Q$-test and $\mathrm{I}^{2}$ index. ${ }^{[14]}$ Second, we performed a multipletreatment meta-analysis using an arm-based, random-effects model within an empirical Bayes framework. ${ }^{[15]}$ The model allowed for estimating effect sizes for all possible pair-wise comparisons of augmentation agents. We also computed the probability that each antidepressant drug was the most efficacious regimen, the second best, the third best, and so on. ${ }^{[16]}$ The ranking of the competing drugs was assessed with the median of the posterior distribution for the rank of each drug. We did the analysis using WinBUGS (Imperial College and MRC, UK) and R version 2.15.0 (R Development Core Team, Austria).

Finally, we looked at comparative efficacy among the augmentation agents. We expressed this using lithium as reference one, because it has been consistently treated as the reference augmentation agent among the different pair-wise comparisons.

\section{Results}

Initial electronic searches (including checking of references of studies) obtained 1905 potentially relevant studies, of which 12 were pooled analyses (Fig. 1). ${ }^{[17-28]}$ All 12 of these studies had obtained ethical approval. Altogether, the 12 studies included 1936 patients 
Search item: depression, augmentation, potentiation, adjunct (up to August 2013)

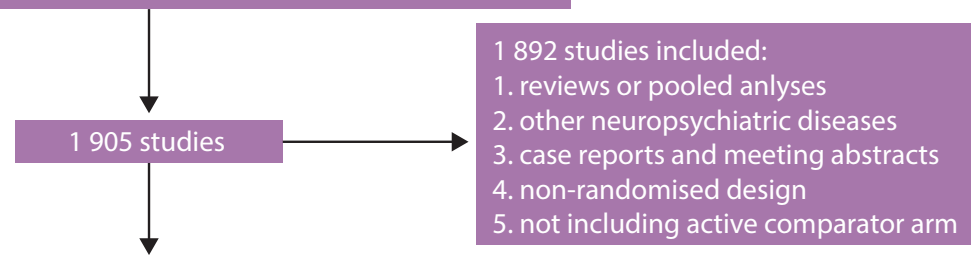

Inclusion criteria:

1. randomised, controlled trial involving augmentation agents

2. patients over 18 years with TRD

3. informed consent provided

4. qualifying score adequate or unclear

5. mood effect assessed by HDRS, MADRS or CGI

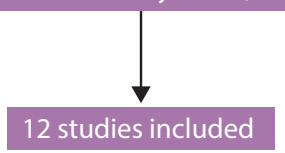

Fig. 1. Literature research. $(\mathrm{TRD}=$ treatment-resistant depression; HDRS = Hamilton Depression Rating Scale; MADRS = Montgomery-Asberg Depression Scale; CGI = Clinical Global Impression.)

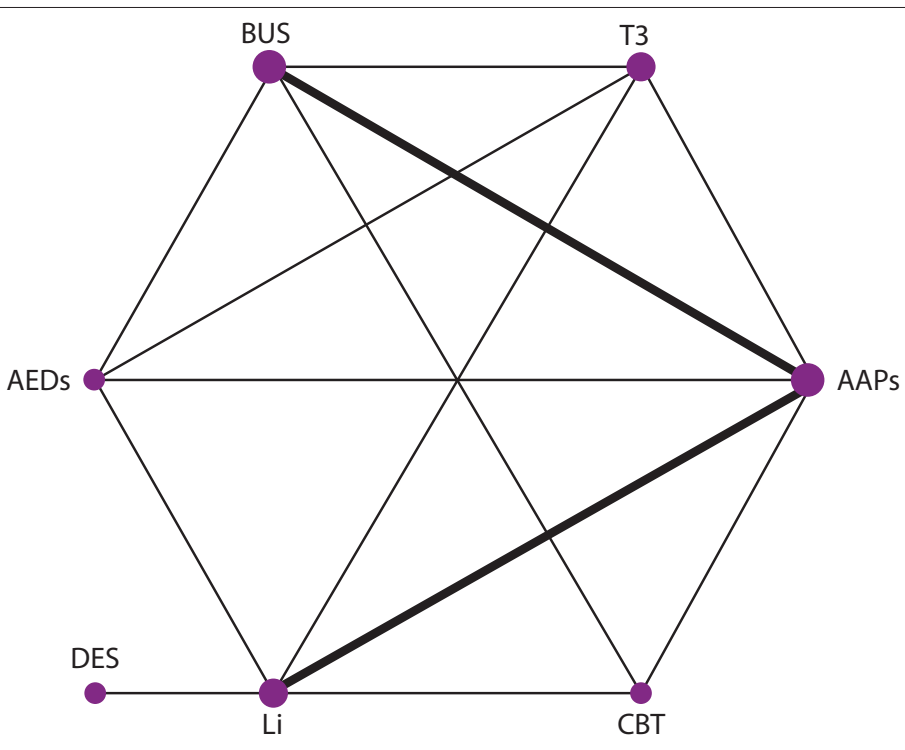

Fig. 2. Network of eligible comparisons for the multiple-treatments meta-analysis. The width of the lines is proportional to the number of patients in each pair of treatments, and the size of each node is proportional to the number of patients received in this treatment. (BUS = buspirone; $\mathrm{T} 3=$ tri-iodothyronine; AAPs = atypical antipsychotics; $\mathrm{CBT}=$ cognitive behaviour therapy; $\mathrm{Li}=$ lithium; TCA = tricyclic antidepressant; AEDs = antiepileptic drugs.)

and seven kinds of augmentation agents, namely lithium, tricyclic antidepressants (TCAs), atypical antipsychotics (AAPs), antiepileptic drugs (AEDs), buspirone, cognitive behaviour therapy (CBT) and tri-iodothyronine (T3). The AAPs in this review included quetiapine, ziprasidone and risperidone. The AEDs included lamotrigine, carbamazepine and sodium valproate. Fig. 2 shows the network of for multiple testing (i.e. approximately two confidence intervals (CIs) would be expected to exclude one by chance alone). For drop-outs, AAPs, AEDs and T3 were better tolerated than lithium, and CBT was better tolerated than AAPs and buspirone. The heterogeneity was moderate for response and drop-out.

Table 2 summarises the results of the multiple-treatments meta-analysis on response (21 simultaneous comparisons). Lithium was non-significantly less effective than all other augmentation agents except buspirone. Buspirone was non-significantly inferior to all other six augmentation agents. CBT was non-significantly superior to all other augmentations except T3. T3 was non-significantly more effective than all other augmentation agents. In terms of acceptability, lithium was less well tolerated than AAPs, AEDs and T3 (Table 3). Analysis indicated no statistical incoherence in any comparisons of direct to indirect evidence for response rate and drop-out rate.

Fig. 3 shows the distribution of probabilities of each augmentation agent being ranked at each of the possible seven positions (ranked number data shown in Table 4). T3 was the most effective augmentation agent, and buspirone was the worst among the seven augmentation agents. The cumulative probabilities of being the most efficacious augmentation agent were: T3 (42.0\%), TCAs (28.7\%), CBT (11.9\%), AEDs (9.6\%), AAPs (4.1\%), buspirone $(2.9 \%)$, lithium (1.0\%).

\section{Discussion}

The results of this review might help clinicians to choose appropriate augmentation agents for acute treatment of TRD. In terms of response, T3 and CBT were more effective than other augmentation agents. Lithium was less effective than the others except buspirone, and buspirone was less effective than all the other augmentation agents. In terms of acceptability, T3 and CBT were comparable to other augmentation strategies. The results indicated that $\mathrm{T} 3$ and $\mathrm{CBT}$ might be favourable options when prescribing an acute treatment for TRD, whereas lithium and buspirone might not. As for TCAs, AAPs and AEDs, further RCTs comparing two or more augmentation agents need to be conducted to assess their relative efficacy and acceptability as augmentation agents.

We retrieved almost all the relevant RCTs on augmentation agents. The missed literature 


\section{ARTICLE}

that were not indexed by international databases were likely to be of low quality, and consequently would not significantly affect the results of this review. ${ }^{[29]}$
T3 has been the second-most investigated augmentation strategy after lithium. One study has suggested that T3 and lithium might be equipotent as augmenters. ${ }^{[30]}$

\begin{tabular}{|c|c|c|c|c|c|}
\hline \multirow{2}{*}{$\frac{\text { Pair }}{1}$} & \multicolumn{2}{|c|}{ Direct comparison } & \multirow{2}{*}{$\begin{array}{l}\text { RR (responders/total) } \\
12 / 48 \text { v. } 13 / 46\end{array}$} & \multirow{2}{*}{$\begin{array}{l}\text { OR } \\
0.85\end{array}$} & \multirow{2}{*}{$\begin{array}{l}\mathbf{9 5 \%} \text { CI } \\
0.34-2.11\end{array}$} \\
\hline & $\mathrm{Li}$ & TCAs & & & \\
\hline 2 & $\mathrm{Li}$ & AAPs & $107 / 231$ v. $124 / 239$ & 0.80 & $0.56-1.15$ \\
\hline 3 & $\mathrm{Li}$ & AEDs & $40 / 90$ v. $36 / 91$ & 1.17 & $0.63-2.19$ \\
\hline 4 & $\mathrm{Li}$ & T3 & $30 / 149$ v. $44 / 160$ & 0.63 & $0.36-1.10$ \\
\hline 5 & $\mathrm{Li}$ & CBT & $10 / 27$ v. $7 / 27$ & 0.84 & $0.24-2.91$ \\
\hline 6 & TCAs & $\mathrm{Li}$ & $13 / 46$ v. $12 / 48$ & 1.18 & $0.47-2.95$ \\
\hline 7 & AAPs & BUS & $21 / 45$ v. $103 / 332$ & 1.95 & $1.04-3.65$ \\
\hline 8 & AAPs & AEDs & $21 / 45$ v. $24 / 39$ & 0.55 & $0.23-1.31$ \\
\hline 9 & AAPs & $\mathrm{T} 3$ & $21 / 45$ v. $28 / 48$ & 0.63 & $0.28-1.42$ \\
\hline 10 & BUS & AEDs & $26 / 46$ v. $24 / 39$ & 0.81 & $0.34-1.94$ \\
\hline 11 & BUS & $\mathrm{T} 3$ & $26 / 46$ v. $28 / 48$ & 0.93 & $0.41-2.10$ \\
\hline 12 & BUS & CBT & $77 / 286$ v. $29 / 85$ & 0.74 & $0.44-1.24$ \\
\hline 13 & AEDs & $\mathrm{Li}$ & $36 / 91$ v. $40 / 90$ & 0.82 & $0.45-1.48$ \\
\hline 14 & AEDs & AAPs & $24 / 39$ v. $21 / 45$ & 1.83 & $0.77-4.37$ \\
\hline 15 & AEDs & BUS & $24 / 39$ v. $26 / 46$ & 1.23 & $0.52-2.94$ \\
\hline 16 & $\mathrm{~T} 3$ & $\mathrm{Li}$ & $44 / 160$ v. $30 / 149$ & 1.50 & $0.89-2.56$ \\
\hline 17 & $\mathrm{~T} 3$ & AAPs & $28 / 48$ v. $21 / 45$ & 1.60 & $0.70-3.63$ \\
\hline 18 & T3 & BUS & $28 / 48$ v. $26 / 46$ & 1.08 & $0.48-2.44$ \\
\hline 19 & CBT & BUS & $29 / 85$ v. $77 / 286$ & 1.36 & $0.81-2.28$ \\
\hline 20 & CBT & $\mathrm{Li}$ & $7 / 27$ v. $10 / 27$ & 0.59 & $0.19-1.90$ \\
\hline
\end{tabular}

$\mathrm{RR}=$ response rate; $\mathrm{OR}=$ odds ratio; $\mathrm{CI}=$ confidence interval; $\mathrm{Li}=$ lithium; TCAs = tricyclic antidepressants; $\mathrm{AAPs}=$ atypical antipsychotics; AEDs = antiepileptic drugs; $\mathrm{T} 3$ = tri-iodothyronine; $\mathrm{CBT}$ = cognitive behaviour therapy; BUS = buspirone.

Another demonstrated T3 to be an efficient augmenter, effective in nearly $60 \%$ of TRD patients, making it a valuable choice in acute-phase treatment of TRD. ${ }^{[28]}$ Owing to its efficacy and acceptability, T3 has been recommended as an alternative augmentation agent. ${ }^{[31]}$ Results of these three studies were consistent with our results, which were that $\mathrm{T} 3$ might be a more effective and acceptable augmentation agent than lithium, TCAs, AAPs, AEDs, buspirone and CBT.

CBT is a type of psychotherapy for the treatment of adults and adolescents with depression. ${ }^{[32]} \mathrm{CBT}$ augmentation therapy has been found to be effective for TRD treatment, ${ }^{[33]}$ and our results indicated that CBT could be a good option. However, it might not be possible to implement CBT augmentation practically owing to lack of CBT-trained clinicians in many communities. It should be considered that the approach might need two separate clinicians: one to provide the psychotherapy and the other to prescribe and monitor the medication treatment. Here, good cooperation between clinicians would be a prerequisite for optimal success.

Lithium is one of the best-studied augmentation therapies in the acute-phase treatment of depressed patients who do not respond to antidepressants. ${ }^{[34]}$ However, owing to a lack of RCTs that directly compared lithium to other augmentation agents

Table 2. Efficacy of seven augmentations*

\begin{tabular}{|c|c|c|c|c|c|c|c|}
\hline \multirow{2}{*}{$\begin{array}{l}\text { Defining augmentation } \\
\mathrm{Li}\end{array}$} & \multirow{2}{*}{ v. } & \multicolumn{6}{|c|}{ Other augmentations, OR (95\% CI) } \\
\hline & & TCAs & AAPs & BUS & AEDs & $\mathrm{T} 3$ & $\mathrm{CBT}$ \\
\hline & & $0.75(0.36-2.08)$ & $0.94(0.64-1.43)$ & $1.02(0.65-1.85)$ & $0.88(0.57-1.51)$ & $0.63(0.35-1.96)$ & $0.87(0.53-1.75)$ \\
\hline \multirow[t]{2}{*}{ TCAs } & & $\mathrm{Li}$ & AAPs & BUS & AEDs & $\mathrm{T} 3$ & $\mathrm{CBT}$ \\
\hline & & $1.33(0.70-1.55)$ & $0.91(0.43-2.85)$ & $1.02(0.44-3.57)$ & $0.86(0.40-2.94)$ & $0.64(0.28-2.12)$ & $0.87(0.37-3.22)$ \\
\hline \multirow{2}{*}{ AAPs } & & $\mathrm{Li}$ & TCAs & BUS & AEDs & T3 & $\mathrm{CBT}$ \\
\hline & & $1.06(0.70-1.55)$ & $1.09(0.35-2.33)$ & $1.12(0.74-1.75)$ & $0.91(0.57-1.72)$ & $0.66(0.43-1.19)$ & $0.97(0.60-1.75)$ \\
\hline \multirow[t]{2}{*}{ BUS } & & $\mathrm{Li}$ & TCAs & AAPs & AEDs & T3 & CBT \\
\hline & & $0.98(0.54-1.55)$ & $0.98(0.28-2.24)$ & $0.89(0.57-1.35)$ & $0.79(0.46-1.58)$ & $0.57(0.33-1.16)$ & $0.81(0.50-1.56)$ \\
\hline \multirow[t]{2}{*}{ AEDs } & & $\mathrm{Li}$ & TCAs & AAPs & BUS & T3 & $\mathrm{CBT}$ \\
\hline & & $1.13(0.66-1.76)$ & $1.16(0.34-2.50)$ & $1.09(0.58-1.75)$ & $1.26(0.63-2.17)$ & $0.68(0.39-1.35)$ & $0.92(0.51-2.12)$ \\
\hline \multirow[t]{2}{*}{ T3 } & & $\mathrm{Li}$ & TCAs & AAPs & BUS & AEDs & $\mathrm{CBT}$ \\
\hline & & $1.58(0.51-2.78)$ & $1.56(0.47-3.52)$ & $1.51(0.84-2.35)$ & $1.77(0.86-3.02)$ & $1.47(0.74-2.57)$ & $1.25(0.69-2.94)$ \\
\hline \multirow[t]{2}{*}{ CBT } & & $\mathrm{Li}$ & TCAs & AAPs & BUS & AEDs & T3 \\
\hline & & $1.14(0.57-1.89)$ & $1.14(0.31-2.65)$ & $1.03(0.59-1.67)$ & $1.23(0.64-2.00)$ & $1.08(0.47-1.97)$ & $0.79(0.34-1.43)$ \\
\hline
\end{tabular}


under similar conditions, whether lithium is better than other augmentation agents or not is largely unknown. Research indicated that there had been no dramatic difference between lithium augmentation and any other augmentation strategies. ${ }^{[35]}$ In this study, we
Table 3. Efficacy and acceptability, using lithium as reference compound

\begin{tabular}{lll}
\hline & Efficacy $^{*}$ (response rate), OR $(\mathbf{9 5 \%} \mathbf{C I})$ & Acceptability $^{\dagger}$ (drop-out rate), OR (95\% CI) \\
\hline TCAs & $1.33(0.48-2.78)$ & $1.93(0.35-5.16)$ \\
AAPs & $1.05(0.73-1.54)$ & $0.23(0.04-0.71)^{\ddagger}$ \\
BUS & $0.98(0.54-1.55)$ & - \\
AEDs & $1.13(0.66-1.76)$ & $0.66(0.08-1.87)$ \\
T3 & $1.33(0.48-2.78)$ & $0.68(0.17-1.67)$ \\
CBT & $1.14(0.57-1.89)$ & - \\
OR = odds ratio; CI = confidence interval; TCAs = tricyclic antidepressants; AAPs = atypical antipsychotics; BUS = buspirone; \\
AEDs = antiepileptic drugs; T3 = tri-iodothyronine; CBT = cognitive behaviour therapy. \\
${ }^{*}$ ORs $<1$ favour lithium. \\
${ }^{\dagger}$ ORs $>1$ favour lithium. \\
${ }^{*} p<0.05$.
\end{tabular}

Table 4. Ranked number of augmentations

\begin{tabular}{llll}
\hline Augmentation & $\mathbf{5 \%} \mathbf{C I}$ & Median & $\mathbf{9 5 \%}$ CI \\
\hline T3 & 3 & 6 & 7 \\
AEDs & 1 & 4 & 6 \\
CBT & 1 & 4 & 7 \\
BUS & 1 & 2 & 6 \\
Li & 1 & 3 & 6 \\
AAPs & 2 & 4 & 6 \\
TCAs & 1 & 5 & 7 \\
CI = confidence interval; T3 = tri-iodothyronine; AEDs = antiepileptic drugs; CBT = cognitive behaviour therapy; \\
BUS = buspirone; Li = lithium; AAPs = atypical antipsychotics; TCAs = tricyclic antidepressants.)
\end{tabular}

found that lithium was non-significantly inferior to other augmentation agents.

Buspirone is an anxiolytic psychoactive drug of the azapirone chemical class and is primarily used to treat generalised anxiety disorder. Although some studies have shown buspirone to be an effective augmentation agent for clinical depression, our results showed that buspirone might not be as effective when compared with other augmentation agents. Moreover, buspirone has many side-effects, which include nervousness, occasional dizziness, restlessness and headache. ${ }^{[36]}$

Several factors might contribute to treatment failure, including misdiagnosed or undiagnosed medical conditions. Therefore, clinicians should first review whether the original diagnosis of depression is correct when patients do not respond or only partially respond to an antidepressant. Also, comorbid disorders, such as substance dependence or substance abuse, might affect treatment response. In addition, poor compliance and adverse effects might be additional obstacles to successful treatment. Therefore, clinicians should take these into consideration before prescribing augmentation therapy.

Most trials included in our meta-analysis did not report adequate information about blinded outcome assessment. This might result in subject bias and undermine the validity of our findings. Nonetheless, in terms of design
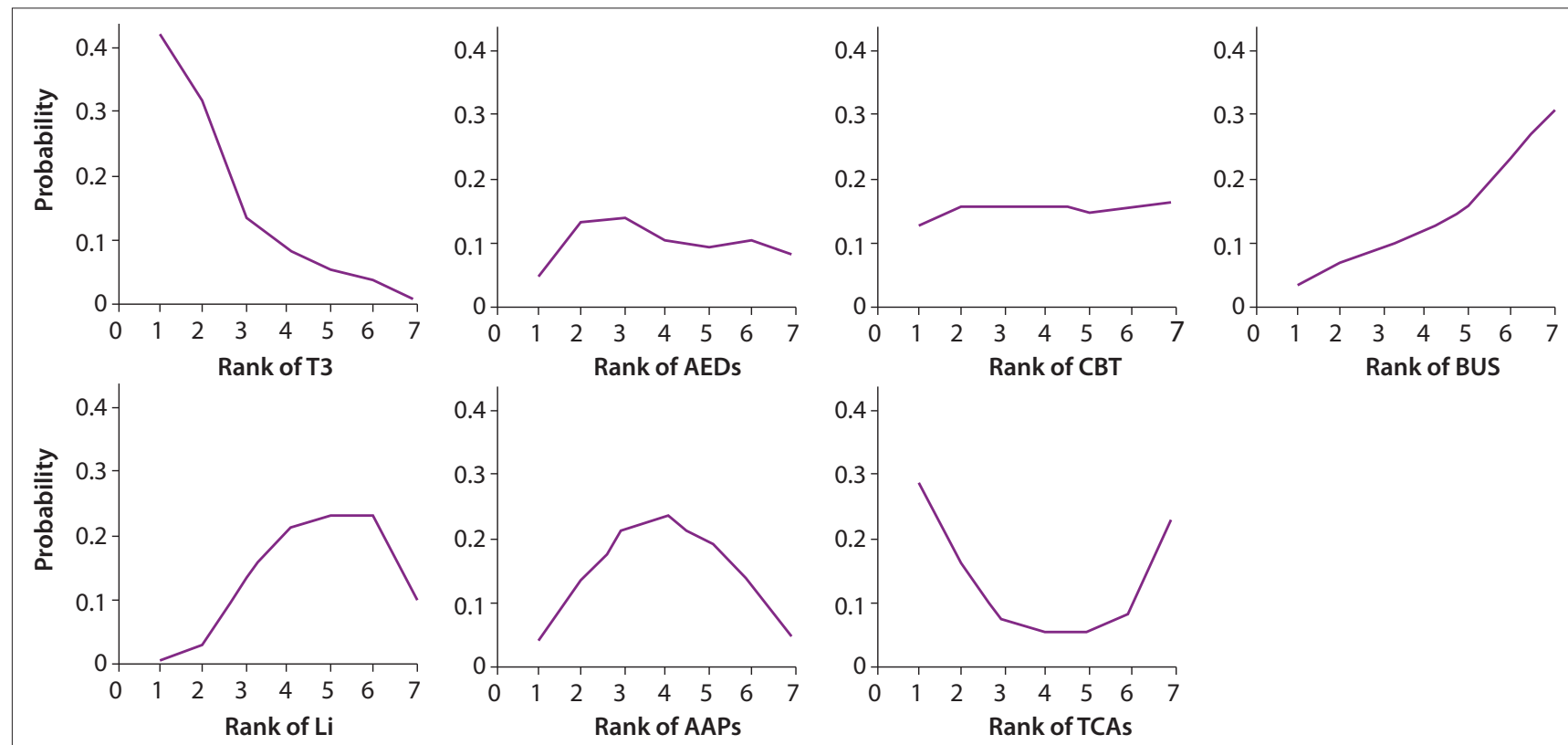

Fig. 3. Ranking for efficacy of seven augmentations. Ranking indicates the probability to be the best treatment, the second best, the third best, and so on. ( $\mathrm{T} 3$ = tri-iodothyronine; AEDs = antiepileptic drugs; $\mathrm{CBT}=$ cognitive behaviour therapy; BUS = buspirone; $\mathrm{Li}=$ lithium; AAPs = atypical antipsychotics; TCAs $=$ tricyclic antidepressants.) 


\section{ARTICLE}
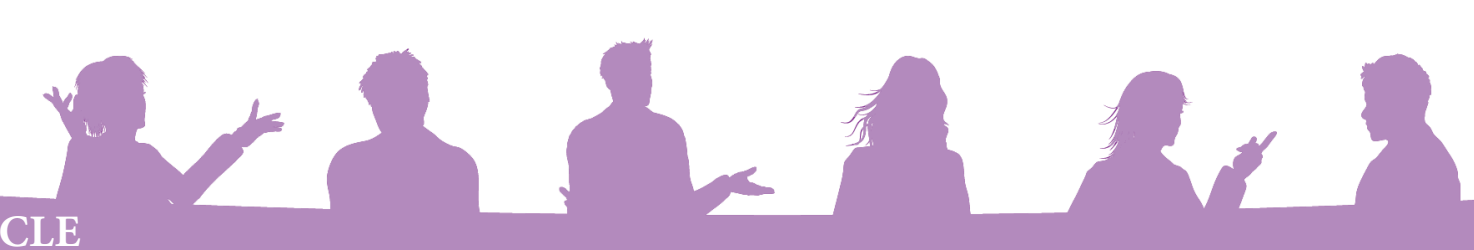

and conduct, all the studies included in this analysis were very similar. Moreover, inadequate information about quality assessment could be a factor of reporting in the text rather than real shortcomings in study design, as has been commonly found in other systematic reviews. ${ }^{[37]}$

\section{Conclusion}

T3, as an augmentation agent, might be clinicians' first consideration instead of lithium in the acute treatment of TRD. CBT might be a good augmentation agent in some communities. Buspirone might be the last option to be considered as an augmentation agent. Because of the limitations of the studies included in this meta-analysis, our conclusions require further research, such as well-designed, large controlled trials.

Acknowledgments. We thank Dr NR Pelliri for his assistance in editing and proofreading the manuscript.

\section{References}

1. Chapman DP, Perry GS. Depression as a major component of public health for older adults. Prev Chronic Dis 2008;5(1):A22.

2. Cipriani A, Furukawa TA, Salanti G, et al. Comparative efficacy and acceptability of 12 new generation antidepressants: A multiple-treatments meta-analysis. Lancet 2009;373(9665):746758. [http://dx.doi.org/10.1016/S0140-6736(09)60046-5]

3. Kroenke K, West SL, Swindle R, et al. Similar effectiveness of paroxetine, fluoxetine, an sertraline in primary care: A randomized trial. JAMA 2001;286(23):2947-2955. [http://dx.doi org/10.1001/jama.286.23.2947]

4. Berlim MT, Turecki G. What is the meaning of treatment resistant/refractory major depression (TRD)? A systematic review of current randomized trials. Eur Neuropsychopharmacol 2007;17(11):696-707. [http://dx.doi.org/10.1016/j.euroneuro.2007.03.009]

5. Bauer M, Whybrow PC, Angst J, Versiani M, Moller HJ. World Federation of Societies of Biological Psychiatry (WFSBP) Guidelines for Biological Treatment of Unipolar Depressive Disorders, Part 1: Acute and continuation treatment of major depressive disorder. World J Bio Psychiatry 2002;3(1):5-43.

6. Carvalho AF, Cavalcante JL, Castelo MS, Lima MCO. Augmentation strategies for treatmentresistant depression: A literature review. J Clin Pharm Ther 2007;32(5):415-428. [http://dx.doi. org/10.1111/j.1365-2710.2007.00846.x]

7. Mischoulon D, Nierenberg AA, Kizilbash L, Rosenbaum JF, Fava M. Strategies for managin depression refractory to selective serotonin reuptake inhibitor treatment: A survey of clinicians. Can J Psychiatry 2000;45(5):476-481.

8. Nelson JC. Treatment of antidepressant non-responders: Augmentation or switch? J Clin Psychiatry 1998;59(Suppl. 15):S35-41.

9. Fava M, Rush AJ. Current status of augmentation and combination treatments for major depressive disorder: A literature review and a proposal for a novel approach to improve practice. Psychother Psychosom 2006;75(3):139-153. [http://dx.doi.org/10.1159/000091771]

10. Thase ME, Rush AJ. When at first you don't succeed: Sequential strategies for antidepressant nonresponders. J Clin Psychiatry 1997;58(Suppl 13):23-29.

11. Chabrol H, Teissedre F, Saint-Jean M, Teisseyre N, Rogé B, Mullet E. Prevention and treatment of post-partum depression: A controlled randomized study on women at risk. Psychol Med 2002;32(6):1039-1047. [http://dx.doi.org/10.1017/S0033291702006062]

12. Guyatt GH, Juniper EF, Walter SD, Griffith LE, Goldstein RS. Interpreting treatment effects in randomised trials. BMJ 1998;316(7132):690-693. [http://dx.doi.org/10.1136 bmj.316.7132.690]

13. Furukawa TA, Cipriani A, Barbui C, Brambilla P, Watanabe N. Imputing response rates from means and standard deviations in meta-analysis. Int Clin Psychopharmacol 2005;20(1):49-52. [http://dx.doi.org/10.1097/00004850-200501000-00010]

14. Higgins JP, Thompson SG, Deeks JJ, Altman DG. Measuring inconsistency in meta-analyses. BMJ 2003:327(7414):557-560. [http://dx.doi.org/10.1136/bmj.327.7414.557]

15. Ades AE, Sculpher M, Sutton A, et al. Bayesian methods for evidence synthesis in costeffectiveness analysis. Pharmacoeconomics 2006;24(1):1-19.
16. Salanti G, Ades AE, Ioannidis JP. Graphical methods and numerical summaries for presenting results from multiple-treatment meta-analysis: An overview and tutorial. J Clin Epidemio 2010;64(2):163-171. [http://dx.doi.org/10.1016/j.jclinepi.2010.03.016]

17. Bauer M, Dellosso L, Kasper S, et al. Extended-release quetiapinefumarate (quetiapine XR) monotherapy and quetiapine XR or lithium as add-on to antidepressants in patients with treatment-resistant major depressive disorder. J Affect Disord 2013;151(1):209-219. [http:// treatment-resistant major depressive
dx.doi.org/10.1016/j.jad.2013.05.079]

18. Dorée JP, Des Rosiers J, Lew V, et al. Quetiapine augmentation of treatment-resistant depression: A comparison with lithium. Curr Med Res Opin 2007;23(2):333-341. [http:// dx.doi.org/10.1185/030079906X162809]

19. Kennedy SH, Segal ZV, Cohen NL, Levitan RD, Gemar M, Bagby RM. Lithium carbonate versus cognitive therapy as sequential combination treatment strategies in partial responder to antidepressant medication: An exploratory trial. J Clin Psychiatry 2003;64(4):439-444 [http://dx.doi.org/10.4088/JCP.v64n0414]

20. Ivković M, Damjanović A, Jovanović A, Cvetić T, Jasović-Gasić M. Lamotrigine versus lithium augmentation of antidepressant therapy in treatment-resistant depression: Efficacy and tolerability. Psychiatria Danubina 2009;21(2):187-193.

21. Nierenberg AA, Fava M, Trivedi MH, et al. A comparison of lithium and T(3) augmentation following two failed medication treatments for depression: A STAR*D report. Am J Psychiatry 2006;163(9):1519-1530. [http://dx.doi.org/10.1176/appi.ajp.163.9.1519]

22. Rybakowski JK, Suwalska A, Chlopocka-Woźniak M. Potentiation of antidepressants with lithium or carbamazepine in treatment-resistant depression. Neuropsychobiology 1999;40(3):134-139. [http://dx.doi.org/10.1159/000026610]

23. Rush AJ, Trivedi MH, Wisniewski SR, et al. Acute and longer-term outcomes in depressed outpatients requiring one or several treatment steps: A STAR D report. Am J Psychiatry 2006;163(11):1905-1917. [http://dx.doi.org/10.1176/appi.ajp.163.11.1905]

24. Schindler F, Anghelescu IG. Lithium versus lamotrigine augmentation in treatmen resistant unipolar depression: A randomized, open-label study. Int Clin Psychopharmaco 2007;22(3):179-182. [http://dx.doi.org/10.1097/YIC.0b013e328014823d]

25. Fava M, Alpert J, Nierenberg A, et al. Double-blind study of high-dose fluoxetine versus lithium or desipramine augmentation of fluoxetine in partial responders and nonresponders to fluoxetine. I Clin Psychopharmacol 2002;22(4):379-387. [http://dx.doi.org/10.1097/00004714-200208000-00008]

26. Fang Y, Yuan C, Xu Y, et al. A pilot study of the efficacy and safety of paroxetine augmented with risperidone, valproate, buspirone, trazodone, or thyroid hormone in adult Chinese patients with treatment-resistant major depression. J Clin Psychopharmacol 2011;31(5):638642. [http://dx.doi.org/10.1097/JCP.0b013e31822bb1d9]

27. Fava M, Rosenbaum JF, McGrath PJ, Stewart JW, Amsterdam JD, Quitkin FM. Lithium an tricyclic augmentation of fluoxetine treatment for resistant major depression: A double-blind controlled study. Am J Psychiatry 1994;151(9):1372-1374.

28. Joffe RT. The use of thyroid supplements to augment antidepressant medication. J Clin Psychiatry 1998;59(Suppl 5):26-29.

29. Deeks JJ, Altman DG, Bradburn MJ. Statistical methods for examining heterogeneity and combining results from several studies in meta-analysis. In: Egger M, Davey Smith G, Altman DG (eds). Systematic Reviews in Health Care: Meta-analysis in Context (2nd ed.). London: BMJ Publication Group, 2001.

30. Joffe RT, Levitt AJ, Bagby RM, C MacDonald, W Singer. Predictors of response to lithium and triiodothyronine augmentation of antidepressants in tricyclic non-responders. Br J Psychiatry 1993;163:574-578. [http://dx.doi.org/10.1192/bjp.163.5.574]

31. Kennedy SH, Lam RW, Cohen NL, Ravindran AV. Clinical guidelines for the treatment of depressive disorders. IV. Medications and other biological treatments. Can J Psychiatry 2001;46(Suppl 1):38S-58S

32. Bridge JA, Iyengar S, Salary CB, et al. Clinical response and risk for reported suicidal ideation and suicide attempts in pediatric antidepressant treatment: A meta-analysis of randomized controlled trials. JAMA 2007:297(15):1683-1696. [http://dx.doi.org/10.1001/jama.297.15.1683]

33. Scott J, Teasdale JD, Paykel ES, et al. Effects of cognitive therapy on psychological symptoms and social functioning in residual depression. Br J Psychiatry 2000;177:440-446. [http:// dx.doi.org/10.1192/bjp.177.5.440]

34. Bauer M, Adli M, Bschor T, et al. Lithium's emerging role in the treatment of refractory majo depressive episodes: Augmentation of antidepressants. Neuropsychobiology 2010;62(1):36-42 [http://dx.doi.org/10.1159/000314308]

35. Jayaraman A, Nandhra H. Systematic review of lithium augmentation in depression resistan to at least two antidepressants. Prim Care Companion J Clin Psychiatry 2009;11(4):172. [http://dx.doi.org/10.4088/PCC.08100677]

36. Scweitzer I, Tuckwell V. Risk of adverse events with the use of augmentation therap for the treatment of resistant depression. Drug Exp 1998;19(6):455-464. [http://dx.doi org/10.2165/00002018-199819060-00003]

37. Huwiler-Müntener K, Jüni P, Junker C, Egger M. Quality of reporting of randomized trials as measure of methodologic quality. JAMA 2002;287(21):2801-2804. [http://dx.doi.org/10.1001/ jama.287.21.2801] 